\title{
SMOQ: a tool for predicting the absolute residue-specific quality of a single protein model with support vector machines
}

Renzhi Cao ${ }^{1 \dagger}$, Zheng Wang ${ }^{2 \dagger}$, Yiheng Wang ${ }^{2}$ and Jianlin Cheng ${ }^{1 *}$

\begin{abstract}
Background: It is important to predict the quality of a protein structural model before its native structure is known. The method that can predict the absolute local quality of individual residues in a single protein model is rare, yet particularly needed for using, ranking and refining protein models.

Results: We developed a machine learning tool (SMOQ) that can predict the distance deviation of each residue in a single protein model. SMOQ uses support vector machines (SVM) with protein sequence and structural features (i.e. basic feature set), including amino acid sequence, secondary structures, solvent accessibilities, and residue-residue contacts to make predictions. We also trained a SVM model with two new additional features (profiles and SOV scores) on 20 CASP8 targets and found that including them can only improve the performance when real deviations between native and model are higher than $5 \AA$. The SMOQ tool finally released uses the basic feature set trained on 85 CASP8 targets. Moreover, SMOQ implemented a way to convert predicted local quality scores into a global quality score. SMOQ was tested on the 84 CASP9 single-domain targets. The average difference between the residue-specific distance deviation predicted by our method and the actual distance deviation on the test data is $2.637 \AA$. The global quality prediction accuracy of the tool is comparable to other good tools on the same benchmark.
\end{abstract}

Conclusion: $\mathrm{SMOQ}$ is a useful tool for protein single model quality assessment. Its source code and executable are available at: http://sysbio.rnet.missouri.edu/multicom_toolbox/.

\section{Background}

With the development of many techniques and tools for protein tertiary structure prediction, a large number of tertiary structure models can be generated for a protein on a computer at a much faster speed than the experimental methods such as X-ray crystallography and nuclear magnetic resonance (NMR) spectroscopy [1,2]. It is becoming increasingly important to develop model quality assessment programs that can predict the qualities of protein models before their corresponding native structures are known, which can help identify quality models or model regions and guide the proper usage of the models [3]. Therefore, the last few rounds of CASP (Critical Assessment of Techniques for Protein Structure Prediction) experiments [4-6]

\footnotetext{
* Correspondence: chengji@missouri.edu

${ }^{\dagger}$ Equal contributors

'Department of Computer Science, Informatics Institute, Christopher S. Bond Life Science Center, University of Missouri, Columbia, MO 65211, USA Full list of author information is available at the end of the article
}

dedicated one model quality assessment (QA) category to specifically evaluate the performances of protein model quality assessment methods, which stimulated the development of such methods and programs in the last several years.

Model quality assessment programs can be categorized into clustering-based methods [7-14], single-model methods [14-18], and hybrid methods [19,20] that combine the previous two. Clustering methods need a set of protein models associated with the same protein sequence as input and can output the relative quality scores by pairwise structural comparison (alignments). Single-model methods only need one model as input and can output the either relative or absolute qualities of the model. In general, clustering-based methods usually had better performances than single-model methods [6,20-22] in the past CASP experiments. However, clustering methods are highly dependent on the size and the quality distribution of the input models. It is hard for them to pick up best models in most cases, especially if the 
best model is not the average model that is most similar to other models. Therefore, it is increasingly important to develop single-model methods that can predict the quality of a single model without referring to any other models.

Model quality assessment programs can either output global quality scores $[11,14,18,23]$ or local quality scores [20,24-27]. A global quality score measures the overall quality of an entire model, whereas local quality scores consisting of a series of scores, one for each residue, measure the quality of the positions of individual residues. For instance, a local quality score may be the predicted distance between the position of residue in a model and that in the native structure after they are superimposed. Because local quality assessment methods can predict residue-specific qualities, it can help identify regions of good quality that can be used or regions of poor quality that needed to be further refined.

Although local quality predictions are very useful, not many local quality assessment methods have been developed. The existent local quality assessment methods mostly use statistical structural environment profiles [26,28-31], energy potentials [32], or pairwise clustering techniques that output relative local qualities [19,33,34]. Verify3D $[29,35]$ is a representative method that compares the structural environment of a query model of a protein with the expected structural profiles for the protein compiled from native protein structures in order to predict the quality of the model. The information that Verify3D used to generate statistical profiles includes secondary structure, solvent accessibility, and residue polarity. ProQres [36] is a machine learning method that uses the structural features calculated from the model with artificial neural networks to predict absolute local qualities.

In this work, we developed and extensively tested a machine learning software tool (SMOQ) that implements a local quality assessment method predicting the absolute local qualities of a single protein model [14]. SMOQ also uses structural features including secondary structure, solvent accessibilities, and residue contact information as input. However, different with Verify3D that directly evaluates the fitness of the structural features parsed from a model, SMOQ compares the structural features parsed from the model with the ones predicted from sequence, and uses the comparison results as input features. In addition to using the features briefly introduced in [14], we tested the effectiveness of new features such as sequence profiles and SOV scores [37] and trained support vector machines on a larger dataset (CASP8) to make predictions. Furthermore, we developed and benchmarked a new method to convert predicted local qualities into a global quality score. Our experiment demonstrated that the global quality scores converted from local quality scores were useful for assessing protein models, particularly the models of hard ab initio targets.

\section{Implementation}

Features for support vector machines (SVM)

We developed and tested three SVM-based predictors using basic, profile, and profile+SOV feature sets respectively. The features in the basic feature set include amino acid sequence, secondary structures, solvent accessibility, and residue-residue contacts. The profile feature dataset is the same as the basic feature set except that amino acid sequence was replaced with sequence profile generated from PSI-BLAST [38]. Compared with the profile feature set, the profile+SOV feature sets added as a feature the SOV (segment overlap measure of secondary structure) scores [37] between the secondary structures predicted from protein sequence and secondary structures parsed from model.

A 15-residue window centered on a target residue in a protein was used to extract features. 20 binary numbers represent an amino acid at each position in the window. We used software SSPRO [39] to predict the secondary structures and solvent accessibility based on the amino acid sequence parsed from each protein model. For each residue position within the window, the predicted secondary structure and relative solvent accessibility were compared with the ones parsed from the protein model by the software DSSP [40]. If they are the same, 1 will be input as a feature for secondary structure or relative solvent accessibility, respectively, otherwise 0 .

We used NNcon [41] to predict the residue-specific contact probability matrix from a protein sequence. For each residue within the 15-residue sliding window, we first used DSSP to parse their coordinates in the models to identify the other residues that are $>=6$ residues away in the sequence and are spatially in contact $(<=8 \AA)$ with the residue. And then we calculated their average predicted probabilities of being contact with the residue according to the contact probability matrix. This averaged value was used as a feature. We calculated the SOV score between the secondary structures predicted from sequence and the secondary structure parsed from model and used it as a feature according to the same approach in [37].

The input features in a window centered at a target residue in a model are used by SVMs to predict the distance deviation between the position of the residue in the model and that in the corresponding native structure. The larger the distance deviation, the lower is the local quality.

\section{Training data set}

Our first training data set contains the complete tertiary structure models of 85 single-domain CASP8 targets (http://predictioncenter.org/casp8/domain_definition.cgi). These targets contain all the single-domain "template based modeling" (53 TBM targets), "template based modelinghigh accuracy" (28 TBM-HA targets), "free modeling" 
(2 FM targets), "free modeling or template based modeling" (2 FM/TBM) targets.

Descriptions about the domain classifications can be found from CASP website (http://predictioncenter.org/ casp8/doc/Target_classification_1.html). For each of these targets, only the first Tertiary Structure (TS) model for a TS predictor was included in our training dataset. These models generated about 600,000 training examples (i.e. feature-distance pairs for the residues in these models) in total. This data set was used to optimize the parameters of the Radial Basis Function (RBF) kernel used with our support vector machines (SVM). A SVM model of using the basic feature set was then trained on this data set using the optimized parameters before being tested on the test data set.

To fairly compare the performances of basic, profile, and profile+SOV feature sets, we also trained them on the same set generated from the protein models associated with the same 20 CASP8 targets. These 20 CASP8 singledomain targets also contain FM, TBM, and TBM-HA targets in a balanced way.

All of the training and testing targets are deliberately chosen to be single-domain proteins. This is because directly superimposing multi-domain model with its native structure often over estimates the distance deviations of residues in individual domains due to possible deviations in domain orientations. An alternative way would be to cut multi-domain models into individual ones and align each domain with its native structure. Since we have a reasonable number of single-domain targets of different modeling difficulty (i.e., TBM, TBM-HA, and FM), we have chosen to only use single-domain targets for training and testing.

\section{Training and cross-validation}

The support vector machine tool SVM-light (http:// svmlight.joachims.org/) was trained on the data set extracted from the CASP8 tertiary structure models. We applied several rounds of 5-folds cross-validation on the training data set. Each round used a different combination of parameters: -c "trade-off between training error and margin", -w "epsilon width of tube for regression", and -g "the gamma parameter in the RBF kernel". The parameter combination that achieved the best performance in a 5-fold cross-validation was finally used to train a SVM model with all the training examples.

\section{Test dataset}

In total, 84 CASP9 single-domain targets were used to blindly benchmark the performances of the QA tools. The tools were tested only using the first TS (tertiary structure prediction) model for each target. Partial TS models that did not have coordinates for all the residues were discarded. In total, $\sim 778,000$ residue-specific local quality examples (data points) were generated as the ground truth to evaluate the local predictions of these

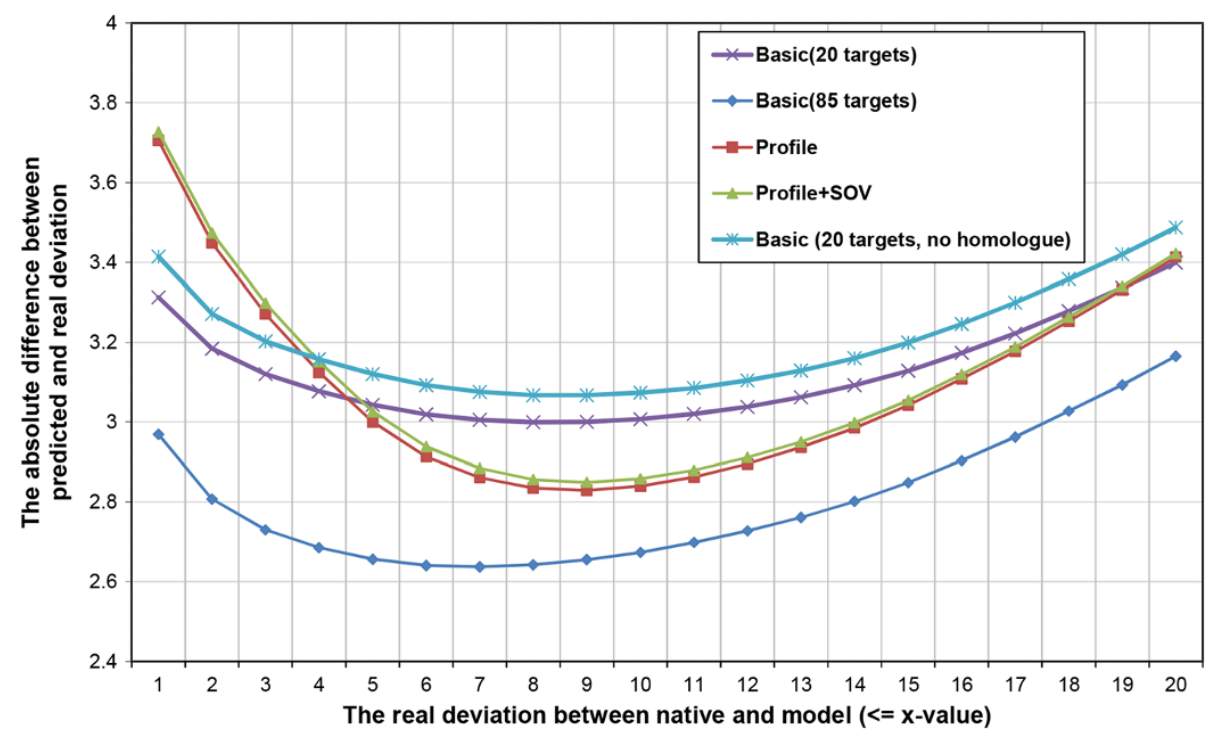

Figure 1 The evaluation results of residue-specific local quality predictions of single-model local quality QA tools (SMOQ) on CASP9 single-domain proteins. Basic (20 targets) denotes the SVM model trained using the basic feature set on 20 CASP8 single-domain targets. Basic (85 targets) denotes the SVM model trained using basic feature set on 85 CASP8 single-domain targets. Basic (20 targets, no homologue) denotes the basic model trained on 20 CASP8 single-domain targets, but tested on the CASP9 single-domain targets that are not homologues of CASP8 targets. Profile and profile+SOV denote the two SVM models using profile and profile+SOV feature set that were trained on 20 CASP8 single-domain targets and tested on CASP9 targets without homologue removal. The absolute difference errors of the predictions were plotted against the real distance deviations. 
Table 1 The average correlation and absolute difference between real and predicted deviation on CASP9 targets for residue-specific quality prediction

\begin{tabular}{lll}
\hline & Avg. correlation & Avg. absolute difference error \\
\hline Basic (85 targets) & 0.42 & 7.09 \\
ProQ2 & 0.47 & 6.63 \\
QMEAN & 0.43 & 7.46 \\
\hline
\end{tabular}

tools. The true global qualities of the models were also used to evaluate the global quality predictions converted from the local quality predictions.

\section{Converting local quality scores into one global quality score}

Based on the local qualities predicted by the local quality predictor trained on the CASP8 data set, we use a variation of Levitt-Gerstein (LG) score [42] to convert the local quality scores into one global quality score for each individual model:

$$
\text { global }=\frac{1}{L} \sum_{i=1}^{L} \frac{1}{1+\left(\frac{d_{i}}{c}\right)^{2}},
$$

where $L$ is the number of amino acid residues in the protein, $d_{i}$ is the predicted distance deviation between the position of residue $i$ in a model and that in the native structure, and $c$ is a constant that was set to 5 in our experiments. This formula was first used by [42] to calculate the similarity score for aligning two protein structures. This formula ensures the global quality remains between $(0,1)$. The parameter $\mathrm{c}$ is a constant, which was set to be $3.5 \AA$ for MaxSub score and $5 \AA$ for the original LG-score and S-score $[42,43]$. Another quality prediction method such as ProQ2 [25] also has used similar approaches to convert local scores into global ones.

\section{Results and discussion}

Benchmarking residue-specific local quality predictions

We trained three different SVM models using three different feature sets ("basic", "profile", and "profile + SOV score") extracted from the CASP8 protein models. Using 778,000 CASP9 local quality examples, we benchmarked and compared the performances of the three QA tools (Figure 1). We used the absolute difference between predicted and real deviation between the position of a residue in a model and that of the same residue in the native structure as a metric to evaluate the predictions. We refer to this metric as absolute difference error. According to Figure 1, as the real distance deviation increases, the absolute difference error of predictions of the three tools decreases at first, reaches the minimum and then increases. The best performance of using the basic feature set happened when the real deviation is $<=7 \AA$, where the absolute distance error is $\sim 2.637 \AA$ for the basic-feature predictor trained on 85 CASP8 targets.

According to the evaluation results in Figure 1, adding profile and profile+SOV feature did not improve the prediction accuracy over the basic feature set for the cases when real distance deviation is $<=5 \AA$. However, when the real deviation is $>5 \AA$, adding profile and profie $+\mathrm{SOV}$ starts to improve prediction accuracy. In general, although the basic feature set trained on 85 CASP8 targets performs better than all others SVM models (trained on 20 CASP8 targets) partially because of the larger training data set, a more extensive training on the same large data set is

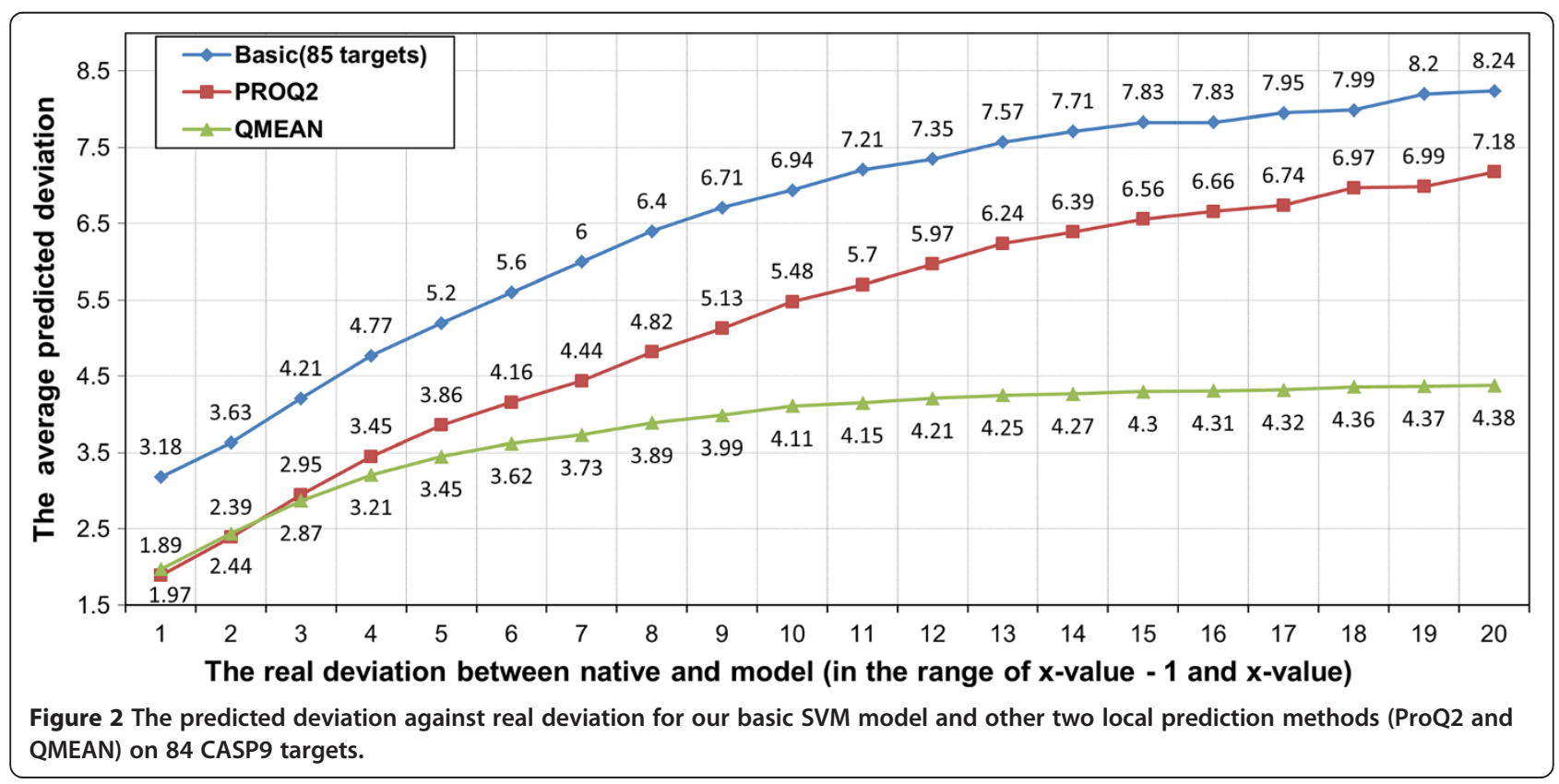




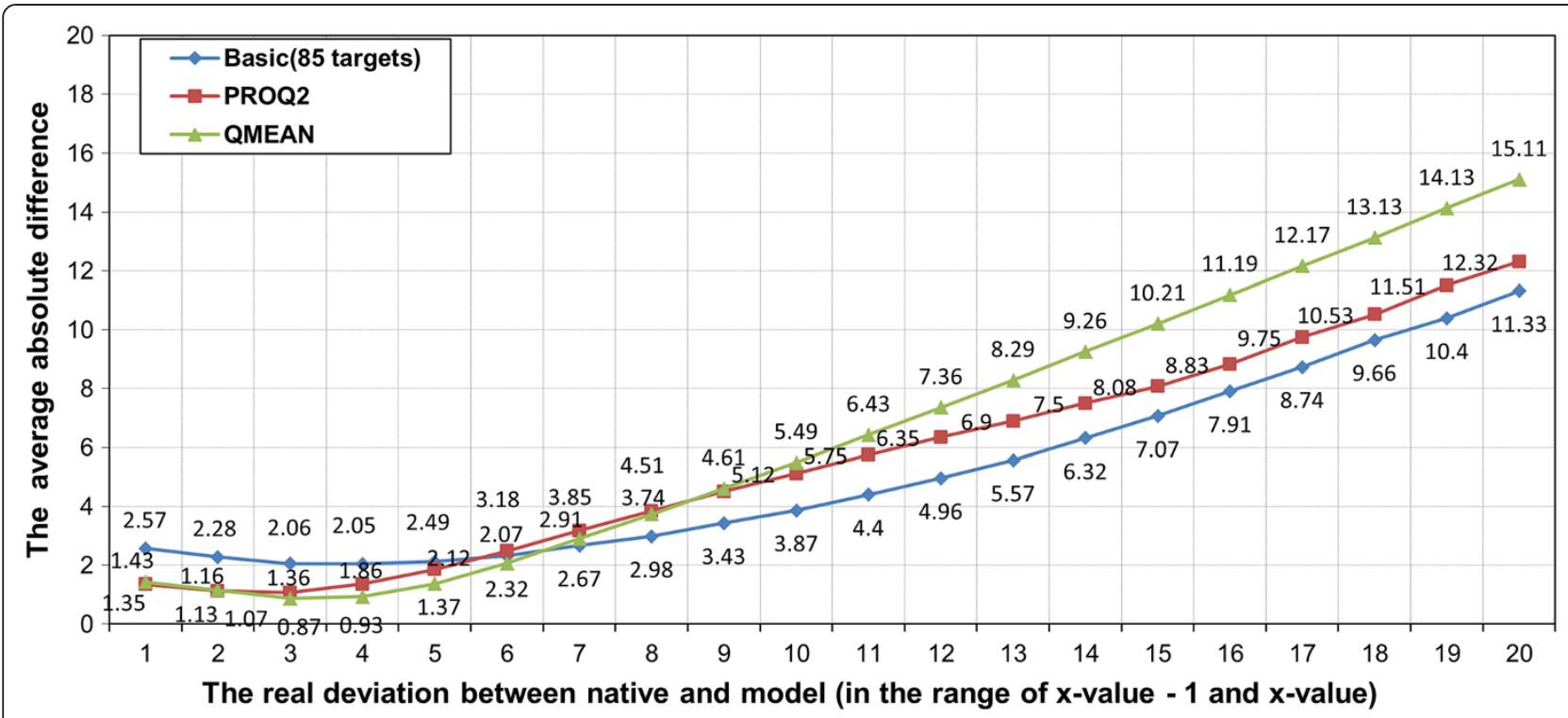

Figure 3 The absolute difference error between real and predicted deviation against real deviation for our basic SVM model and ProQ2 and QMEAN.

needed in order to more rigorously compare the performance of the feature sets with or without profile and SOV features. The SMOQ tool that we finally released was trained on 85 CASP8 targets using the basic feature set.

We trained the SVM models on CASP8 targets and benchmarked them on CASP9 targets, which contain some homologues of CASP8 targets. Therefore, we also eliminated all the CASP9 targets that are significant homologue to CASP8 targets according to PSI-BLAST comparison and used the remaining CASP9 targets to benchmark the performance of the basic-feature predictor trained on 20 CASP8 targets (see Figure 1). The performance is about $0.1 \AA$ worse than without removing homologues.

The average absolute difference error and average correlation coefficient on all CASP9 examples were reported in Table 1. The average correlation of our basic SVM model trained on 85 CASP8 targets is somewhat lower than ProQ2, but very close to QMEAN. Our basic SVM model performs better than QMEAN in terms of average absolute difference error, but worse than ProQ2. Figure 2 plots the average absolute difference error with respect to different real deviations. Our basic SVM model has higher absolute difference error than ProQ2 or QMEAN for the cases when real deviation is $<=6 \AA$, but for cases whose real deviation is $>=7 \AA$, our basic SVM model has lower absolute difference error.

Figure 3 shows the relationship between real and predicted distance deviation for basic, ProQ2, and QMEAN. We noticed that QMEAN tends to predict smaller values for deviation when the real deviation actually is large. For example, the predicted deviation remains between 4 to

Table 2 The performance of the global quality predictions of our three tools and the other four methods in terms of average correlation, overall correlation, average real GDT-TS score of top 1 models ranked by each method, and average loss of top 1 models ranked by each method, evaluated on 84 CASP9 single-domain targets

\begin{tabular}{lllll}
\hline & Avg. correlation & Over. correlation & Avg. top 1 & Avg. loss \\
\hline Basic (85 targets) & $\mathbf{0 . 7 3 7}$ & 0.737 & 0.588 & 0.082 \\
Profile & $\mathbf{0 . 7 0 8}$ & 0.658 & 0.589 & 0.080 \\
Profile+SOV & 0.696 & 0.681 & 0.594 & $\mathbf{0 . 0 7 5}$ \\
ModelEvaluator & 0.636 & $\mathbf{0 . 7 6 7}$ & $\mathbf{0 . 5 9 7}$ & $\mathbf{0 . 0 7 3}$ \\
ProQ & 0.494 & 0.707 & 0.563 & 0.110 \\
ProQ2 & 0.662 & $\mathbf{0 . 7 8 7}$ & $\mathbf{0 . 6 0 7}$ & $\mathbf{0 . 0 6 6}$ \\
QMEAN & $\mathbf{0 . 7 3 3}$ & $\mathbf{0 . 8 0 3}$ & $\mathbf{0 . 5 9 4}$ & 0.078 \\
\hline
\end{tabular}

Basic, profile, and profile + SOV are the three single-model local QA tools (SMOQ) presented in this manuscript.

The other four QA predictors are ModelEvaluator (predictor name in CASP9: MULTICOM-NOVEL), ProQ, ProQ2, and QMEAN. Top 3 QA predictors' performances according to each metric were bolded. 
Table 3 The performance of the QA predictor in terms of average correlation, overall correlation, average real GDT-TS score of top 1 models ranked by each method, and average loss of top 1 models ranked by each method, evaluated on 8 FM (free modeling) CASP9 single-domain targets

\begin{tabular}{lllll}
\hline & Avg. correlation & Over. correlation & Avg. top 1 & Avg. loss \\
\hline Basic (85 targets) & $\mathbf{0 . 5 7 7}$ & $\mathbf{0 . 5 1 6}$ & $\mathbf{0 . 2 6 7}$ & $\mathbf{0 . 0 7 8}$ \\
Profile & $\mathbf{0 . 5 9 0}$ & 0.427 & 0.254 & 0.091 \\
Profile + SOV & $\mathbf{0 . 5 8 6}$ & 0.431 & $\mathbf{0 . 2 6 7}$ & $\mathbf{0 . 0 7 8}$ \\
M.-NOVEL & 0.386 & $\mathbf{0 . 4 8 0}$ & 0.235 & 0.115 \\
ProQ & 0.478 & 0.437 & 0.266 & 0.090 \\
ProQ2 & 0.529 & $\mathbf{0 . 4 6 5}$ & $\mathbf{0 . 2 8 9}$ & $\mathbf{0 . 0 6 6}$ \\
QMEAN & 0.507 & 0.456 & 0.266 & 0.090 \\
\hline
\end{tabular}

Top 3 QA predictors' performances according to each metric were bolded.

$4.4 \AA$ when the real deviation increases from 10 to $20 \AA$. Overall, our SVM model's performance is somehow comparable to ProQ2 or QMEAN. And our method seems to be complementary with ProQ2 and QMEAN.

\section{Benchmarking global quality predictions converted from} local quality predictions

Based on the residue-specific local quality predictions, we generate absolute global qualities for each TS model. We benchmarked and compared the performance of our local to global quality predictions with the other four single-model global quality prediction tools including ModelEvaluator [18], ProQ [17], ProQ2 [25], and QMEAN [16]. It is worth noting that we only evaluated the performance of these methods on the CASP9 single-domain targets rather than all the kinds of protein targets in order to gauge the accuracy and correctness of our tool. A complete and comprehensive assessment of the other methods can be found in the CASP9 quality assessment paper [44].
Table 2 shows the performances of the QA predictors in terms of average correlation (the average per-target correlation between predicted and real quality scores of the models of each protein target), overall correlation (the correlation between predicted and real quality scores of all the models of all the targets), the average real GDT-TS score of top one models for the targets ranked by each QA predictor, and average loss (the average difference between the GDT-scores of the really best models and those of the top 1 models ranked by each predictor), evaluated on 84 CASP9 single-domain targets. Table 3 reports the performances of the same predictors on eight free modeling (FM) CASP9 single-domain targets.

It is shown that our predictors using basic/profile features achieved the best or second performances in terms of the average correlation metric (Table 2), which was the official criterion used in the CASP experiment. Our tools also achieved descent, but not the top performance according to other criteria (Table 2). The performance of
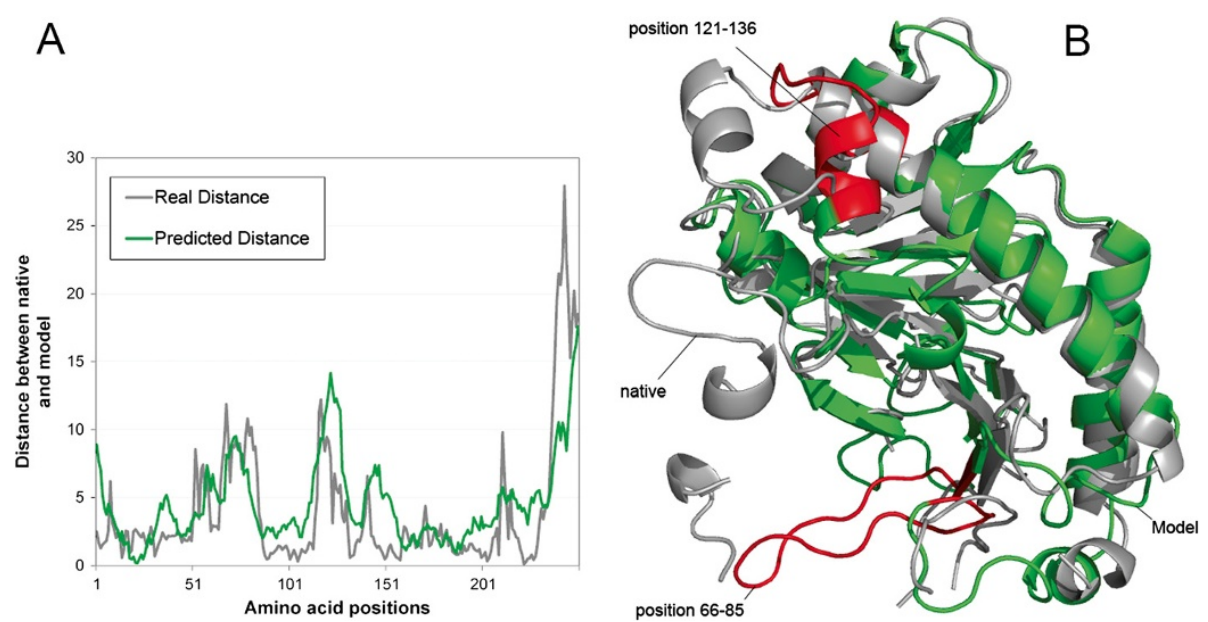

Figure 4 An example illustrates the real and predicted distances between a model and the native structure. The model is the first model of the MULTICOM-CLUSTER tertiary structure predictor for CASP9 target T0563. (A) The real and predicted distance between the native structure and the model at each amino acid position. (B) The superimposition between the model (green and red) and the native structure (grey). Red highlights the two regions where the model has a relatively large deviation compared with the native structure. 
our tools on the free modeling (or $a b$ initio) targets was even better. The models for the free model targets were generated by ab initio protein structure predictors, whose quality was generally much worse than models constructed from known homologous template structures. Thus, it is harder to predict the quality of models of free modeling targets. Table 3 shows that our tool using the basic feature set was constantly ranked within top three. The tool using profile and profile $+\mathrm{SOV}$ achieved better performances than the one using basic feature set in terms of the average correlation criteria. Overall, the global quality prediction performance of our tools on the CASP9 single-domain targets is comparable to the best single-model quality predictors.

\section{An example of local quality predictions}

Figure 4 illustrates a good example of using our tool based on the basic feature set to predict the local qualities of a model [45] in CASP9. The average difference between real and predicted distance deviation is $2.38 \AA$. This model (green) contains two regions with a relatively large distance deviation with the native structure. One region contains a short helix and the other is a loop. These two regions were highlighted in red in Figure 4 (B). Correspondingly, in Figure 4 (A) the two peaks indicating the larger distance deviation were predicted for these two regions.

\section{Conclusions}

We developed and tested the single-model local quality assessment tools (SMOQ) that can predict the residuespecific absolute local qualities of a single protein model. SMOQ is different from the majority of model quality assessment programs in terms of both methodologies and output. The predicted local qualities were also converted into one single score to predict the global quality of a model. The SMOQ tools were rigorously tested on a large benchmark and yielded a performance comparable to other leading methods. However, in this work, we only used single-domain CASP8 targets for training. In the future, we plan to include multi-domain targets by cutting a whole multi-domain model into individual domains and only aligning each domain with its native structure to generate real local quality scores for training. Another future work is to test other functions of converting local scores into global ones. Overall, we believe that SMOQ is a useful tool for both protein tertiary structure prediction and protein model quality assessment.

\section{Availability and requirements}

Project name: SMOQ

Project homepage: http://sysbio.rnet.missouri.edu/multicom_toolbox/

Operating systems: Linux
Programming language: Perl

Other requirements: no

License: Free academic usage

Any restrictions to use by non-academics: For nonacademic use, please contact the corresponding author for permission

Competing interests

The authors declare that they have no competing interests.

\section{Authors' contributions}

JC conceived the project. JC, ZW, RC designed the project. ZW, RC, and YW implemented and tested the tool. RC, ZW, JC prepared the software package. ZW, RC, JC wrote the manuscript. All the authors read and approved the manuscript.

Acknowledgements

This work is partially supported by NIH R01 (R01GM093123) grant to JC.

\section{Author details}

'Department of Computer Science, Informatics Institute, Christopher S. Bond Life Science Center, University of Missouri, Columbia, MO 65211, USA. ${ }^{2}$ School of Computing, University of Southern Mississippi, Hattiesburg, MS 39406-0001, USA.

Received: 17 December 2013 Accepted: 15 April 2014 Published: 28 April 2014

\section{References}

1. Jaravine $V$, Ibraghimov I, Orekhov V: Removal of a time barrier for high-resolution multidimensional NMR spectroscopy. Nat Methods 2006, 3(8):605-607.

2. Lattman $\mathrm{E}$ : The state of the protein structure initiative. Protein Struct Funct Bioinformatics 2004, 54(4):611-615.

3. Baker D, Sali A: Protein structure prediction and structural genomics. Science 2001, 294(5540):93-96.

4. Kryshtafovych A, Fidelis K, Moult J: Progress from CASP6 to CASP7. Protein Struct Funct Bioinformatics 2007, 69(S8):194-207.

5. Moult J, Fidelis K, Kryshtafovych A, Rost B, Hubbard T, Tramontano A: Critical assessment of methods of protein structure prediction-Round VII. Protein Struct Funct Bioinformatics 2007, 69(S8):3-9.

6. Cozzetto D, Kryshtafovych A, Tramontano A: Evaluation of CASP8 model quality predictions. Protein Struct Funct Bioinformatics 2009, 77(S9):157-166.

7. McGuffin L: Benchmarking consensus model quality assessment for protein fold recognition. BMC Bioinforma 2007, 8:345.

8. McGuffin L: The ModFOLD server for the quality assessment of protein structural models. Bioinformatics 2008, 24(4):586.

9. McGuffin L, Roche D: Rapid model quality assessment for protein structure predictions using the comparison of multiple models without structural alignments. Bioinformatics 2010, 26(2):182-188.

10. Paluszewski $M$, Karplus $K$ : Model quality assessment using distance constraints from alignments. Proteins 2008, 75:540-549.

11. Wallner B, Elofsson A: Prediction of global and local model quality in CASP7 using Pcons and ProQ. Proteins 2007, 69(8):184-193.

12. Zhang Y, Skolnick J: SPICKER: a clustering approach to identify nearnative protein folds. J Comput Chem 2004, 25(6):865-871.

13. Ginalski K, Elofsson A, Fischer D, Rychlewski L: 3D-Jury: A Simple Approach to Improve Protein Structure Predictions. Bioinformatics 2003, 19(8):1015-1018.

14. Wang Z, Eickholt J, Cheng J: APOLLO: a quality assessment service for single and multiple protein models. Bioinformatics 2011, 27(12):1715-1716.

15. Archie J, Karplus K: Applying undertaker cost functions to model quality assessment. Proteins 2009, 75:550-555.

16. Benkert P, Tosatto S, Schomburg D: QMEAN: A comprehensive scoring function for model quality assessment. Proteins: Structure, Function, and Bioinformatics 2008, 71(1):261-277.

17. Wallner B, Elofsson A: Can correct protein models be identified? Protein Sci 2003, 12(5):1073-1086.

18. Wang Z, Tegge A, Cheng J: Evaluating the absolute quality of a single protein model using structural features and support vector machines. Proteins 2008, 75:638-647. 
19. Cheng J, Wang Z, Tegge AN, Eickholt J: Prediction of global and local quality of CASP8 models by MULTICOM series. Proteins 2009, 77(S9):181-184.

20. McGuffin L: Prediction of global and local model quality in CASP8 using the ModFOLD server. Protein Struct Funct Bioinformatics 2009, 77(S9):185-190.

21. Kryshtafovych A, Krysko O, Daniluk P, Dmytriv Z, Fidelis K: Protein structure prediction center in CASP8. Proteins 2009, 77(Suppl 9):000-000

22. Larsson P, Skwark MJ, Wallner B, Elofsson A: Assessment of global and local model quality in CASP8 using Pcons and ProQ. Proteins 2009, 77(S9):167-172.

23. Benkert $P$, Kunzli $M$, Schwede $T$ : QMEAN server for protein model quality estimation. Nucleic Acids Res 2009, 37(suppl 2):W510.

24. Wang Z, Tegge AN, Cheng J: Evaluating the absolute quality of a single protein model using structural features and support vector machines. Protein Struct Funct Bioinformatics 2009, 75(3):638-647.

25. Ray $A$, Lindahl $E$, Wallner $B$ : Improved model quality assessment using ProQ2. BMC Bioinforma 2012, 13(1):224.

26. Fasnacht $M$, Zhu J, Honig B: Local quality assessment in homology models using statistical potentials and support vector machines. Protein Sci 2007, 16(8):1557-1568.

27. Sims $\mathrm{GE}, \mathrm{Kim} \mathrm{S}-\mathrm{H}$ : A method for evaluating the structural quality of protein models by using higher-order $\varphi-\psi$ pairs scoring. Proc Natl Acad Sci $U$ S A 2006, 103(12):4428-4432.

28. Luethy R, Bowie J, Eisenberg D: Assessment of protein models with threedimensional profiles. Nature 1992, 356(6364):83-85.

29. Eisenberg D, Luthy R, Bowie J: VERIFY3D: assessment of protein models with three-dimensional profiles. Methods Enzymol 1997, 277:396-404

30. Sippl M: Recognition of errors in three-dimensional structures of proteins. Proteins 1993, 17:355-362.

31. Colovos C, Yeates T: Verification of protein structures: patterns of nonbonded atomic interactions. Protein Sci 1993, 2(9):1511

32. Maiorov V, Abagyan R: Energy strain in three-dimensional protein structures. Fold Des 1998, 3(4):259-269.

33. Benkert P, Schwede T, Tosatto SCE: QMEANclust: estimation of protein model quality by combining a composite scoring function with structural density information. BMC Struct Biol 2009, 9:35.

34. Melo F, Feytmans E: Assessing protein structures with a non-local atomic interaction energy1. J Mol Biol 1998, 277(5):1141-1152.

35. Bowie J, Luthy $R$, Eisenberg D: A method to identify protein sequences that fold into a known three-dimensional structure. Science 1991, 253(5016):164-170.

36. Wallner B, Elofsson A: Identification of correct regions in protein models using structural, alignment, and consensus information. Protein Sci 2006, 15(4):900-913.

37. Zemla A, Venclovas C, Fidelis K, Rost B: A modified definition of Sov, a segment based measure for protein secondary structure prediction assessment. Protein Struct Funct Bioinformatics 1999, 34(2):220-223.

38. Altschul SF, Madden TL, Schäffer AA, Zhang J, Zhang Z, Miller W, Lipman DJ: Gapped BLAST and PSI-BLAST: a new generation of protein database search programs. Nucleic Acids Res 1997, 25(17):3389-3402.

39. Cheng J, Randall AZ, Sweredoski MJ, Baldi P. SCRATCH: a protein structure and structural feature prediction server. Nucleic Acids Res 2005, 33(Web Server Issue):W72-W76.

40. Kabsch W, Sander C: Dictionary of protein secondary structure: pattern recognition of hydrogen-bonded and geometrical features. Biopolymers 1983, 22(12):2577-2637.

41. Tegge AN, Wang Z, Eickholt J, Cheng J: NNcon: improved protein contact map prediction using 2D-recursive neural networks. Nucleic Acids Res 2009, 37(suppl 2):W515.

42. Levitt $M$, Gerstein M: A unified statistical framework for sequence comparison and structure comparison. Proc Natl Acad Sci 1998, 95(11):5913-5920.
43. Zhang Y, Skolnick J: Scoring function for automated assessment of protein structure template quality. Protein Struct Funct Bioinformatics 2004, 57(4):702-710.

44. Kryshtafovych A, Fidelis K, Tramontano A: Evaluation of model quality predictions in CASP9. Protein Struct Funct Bioinformatics 2011, 79(S10):91-106.

45. Wang Z, Eickholt J, Cheng J: MULTICOM: a multi-level combination approach to protein structure prediction and its assessments in CASP8. Bioinformatics 2010, 26(7):882-888.

doi:10.1186/1471-2105-15-120

Cite this article as: Cao et al:: SMOQ: a tool for predicting the absolute residue-specific quality of a single protein model with support vector machines. BMC Bioinformatics 2014 15:120.

\section{Submit your next manuscript to BioMed Central and take full advantage of:}

- Convenient online submission

- Thorough peer review

- No space constraints or color figure charges

- Immediate publication on acceptance

- Inclusion in PubMed, CAS, Scopus and Google Scholar

- Research which is freely available for redistribution

Submit your manuscript at www.biomedcentral.com/submit
C) Biomed Central 